\title{
Land Mobile GNSS Availability and Multipath Evaluation Tool
}

\author{
Juliette Marais, Marion Berbineau, Marc Heddebaut
}

\begin{abstract}
Applications of GNSS (Global Navigation Satellite System) in land transportation systems are already extensively deployed and will certainly continue to grow especially in the framework of Intelligent Transport Systems. However, one of the best-known drawbacks of such a system is the lack of satellite visibility in dense urban areas as well as in some specific embedded railway environments. This restricts considerably GNSS use for extended safety related applications. In this paper, a new tool is proposed to predict the availability of a satellite constellation from the point of view of the land transportation user. Knowing the trajectory of a land vehicle, the tool predicts the number of satellites which will be received and produces a safety criterion able to qualify the GNSS localization result. A first version of the tool, already in operation, merges an image processing approach providing the knowledge of the land environment, and the output of a satellite tracking program predicting satellite positions in the sky. This allows us to determine, using a simple optical approach, the number of satellites received in line-of-sight or blocked, with regard to the nearby environment of the receiving antenna. Results obtained in railway as well as in road environments show that satellite signals received by multipath are often used by GNSS receivers in the localization process. Thus, propagation characteristics of the satellite signals in an urban canyon configuration were characterized to determine when a signal received by multipath is used by the receiver or not. A criterion related to the satellite elevation is defined to improve the overall performance of the predictive tool. Comparisons with real measurements are commented on. Both simulations and measurements are very similar.
\end{abstract}

Index Terms - Localization, satellites, multipath, availability, radio electric coverage.

\section{INTRODUCTION}

M ost of the applications for localization and navigation in the field of transport use satellite systems (such as GPS, GLONASS, or the future European GALILEO system) [1][2]. They are now widespread all over the world. They have been generally developed for road applications. Some are easily transposable in a railway environment for non-safety

Manuscript received June xx, 2002. This work was supported under contract with the French government and european projects.

The authors are with the French National Institute for Transport and Safety Research, Laboratory of Transport Electronics and Signals Processing, 59650 Villeneuve d'Ascq. applications (fleet management...) [3]. Nevertheless, the development of critical applications concerning safety such as railway control and command, relies on the accurate determination of many parameters for which system availability is a crucial point [4][5]. Furthermore, there is a growing need to develop low-cost, safe localization and navigation systems, such as the core of a train commandcontrol system on low density railway lines, or for automated highway applications.

The performances and reliability of the localization system are strongly related to parameters such as localization accuracy, receiver acquisition rate, availability and integrity of the signals. The overall availability of the system is defined as the percentage of time that the services of the system are usable [6]. For satellite-based systems, the availability depends on several criteria such as the number of visible satellites, their relative position within the constellation, the radio electric coverage of the constellation and the health of these satellites which have an impact on the integrity of the signals. Most of the localization and navigation systems in operation today compensate the lack of availability of the satellite constellation [7][8][9] by adding so called "augmentation" solutions. This consists of a sensor fusion approach [10] between the information given by the satellite receiver and others such as broadcasting of local geographic information, map matching [11][12], analysis of mechanical information (gyroscope, speedometer...) [13] and sometimes information given by terrestrial localization beacons judiciously deployed along the vehicle trajectory. For the development of safe localization applications for road and railway transport, it is in particular necessary to be able to predict the availability of the satellite constellation with an evaluation tool in order to simulate the overall system. The major goal of such a tool would be to provide system safety demonstrations with accurate availability parameters.

This paper reports on a predictive tool based on image processing techniques associated with a satellite trajectography software. It is organized as follows : section II gives the principles of the tool. A single run along the vehicle trajectory is necessary in order to record the images of the close vehicle environment as seen by the on-board receiving antenna. The results obtained are presented in section III. They show the interest of the method but also, in order to 
refine the tool, the necessity to take into account the propagation phenomena encountered by the signal in the very last part of its trip. The characterization of the propagation effects on the satellite signals and particularly the impact of multipath on the performances of the localization system are detailed in section IV. The theoretical and experimental approaches are presented. The results and enhancements of the tool are reported in section V.

\section{PRINCIPLES OF THE TOOL}

A method to predict the availability of a satellite system along a fixed trajectory could be simply to run a large number of times along this railway line or road in order to compute statistical values of the GPS satellite visibility as a function of time and space. This method needs a huge quantity of data particularly in the case of non geostationary constellations and is obviously time consuming.

The PREDISSAT tool (PREDIctive Software for Satellite Availability in the field of Transport) developed at INRETS and presented in this paper allows us to determine the number of satellites which will be received and available for localization, at any time and anywhere along a given trajectory. The tool includes a simulation stage for the knowledge of the satellite positions relying on the STK (Satellite Tool Kit) software [14] and an image-processing stage which analyzes a video record of the optical environment surrounding the receiving antenna associated with a mono-camera stereovision process in order to extract distance and heights of the principle obstacles surrounding the vehicle. This is performed in order to extract the satellites which could be received effectively.

The GPS system is a very well-known MEO (Medium Earth Orbit) satellite constellation offering worldwide coverage. The localization process of the GPS system is based on the TOA (Time Of Arrival) method. When no other information is added, a minimum of four satellites is needed to compute an observer position. The best results on time measurement are theoretically obtained with satellites that are geometrically distant from each others providing a line-ofsight (LOS) radio link. This refers to the parameter called Position Dilution of Precision (PDOP) that quantifies the geometrical precision of the localization result. The lower the PDOP, the better the computation accuracy. Several phenomena corrupt the result of the localization : propagation delays in the atmosphere, clock bias, and multipath propagation generate errors in the receiver-to-satellite distance evaluation.

GPS receivers track several satellites simultaneously with trade-offs between satellites received only in LOS with a high PDOP and those received due to multipath which introduces error in the satellite-vehicle pseudo-range.

A simple optical approach illustrated in Fig. 1 allows us to define an "optical horizon line" such that a satellite is "visible" above it, and "blocked" under it. Nevertheless, satellites can be received without direct ray. The satellite elevation is then under the optical mask horizon line and the signal is received by multipath. As suggested in [15]-[18], it is then possible to classify the satellite signal into three states: LOS, multipath with no direct ray, or blocked.

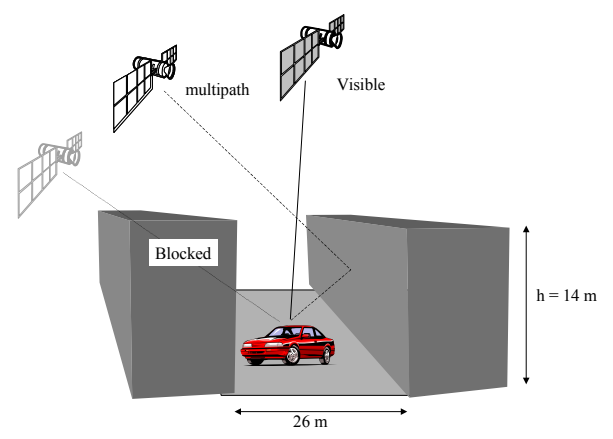

Fig. 1. Illustration of the optical visibility.

A preliminary version of the tool identified only the satellites received in LOS or blocked. The "optical horizon line" is defined as the limit between the obstacles and the sky. The nature of the obstacles is not taken into account. It is automatically detected by the image processing part of the tool on the recorded images along the vehicle trajectory as illustrated in Fig. 2. The elevations and azimuths of the masks around each point of the trajectory are then computed.

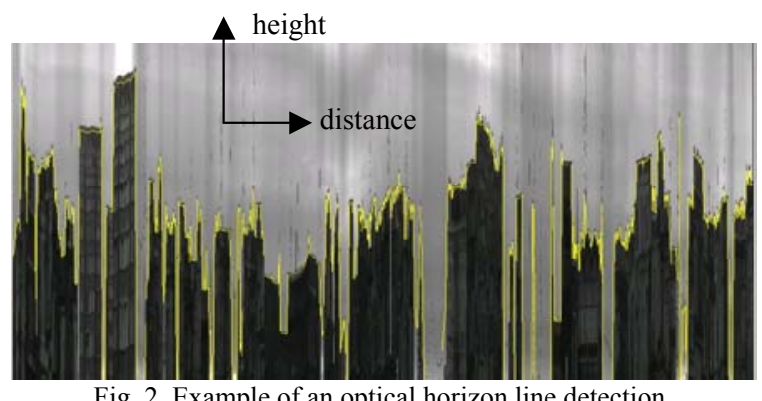

The elevations and azimuths of the satellites are given by STK. The PREDISSAT tool compares satellite positions with positions and elevations of the masks. For example, a schematic view of the process on a point of the trajectory is given in Fig. 3. The graph on the left represents the position of 5 satellites around the antenna. The right one shows, from the same antenna point, the elevation angles all around the antenna. The intersection of both graphs determines whether each satellite is over or under the mask. The tool is detailed in [19].
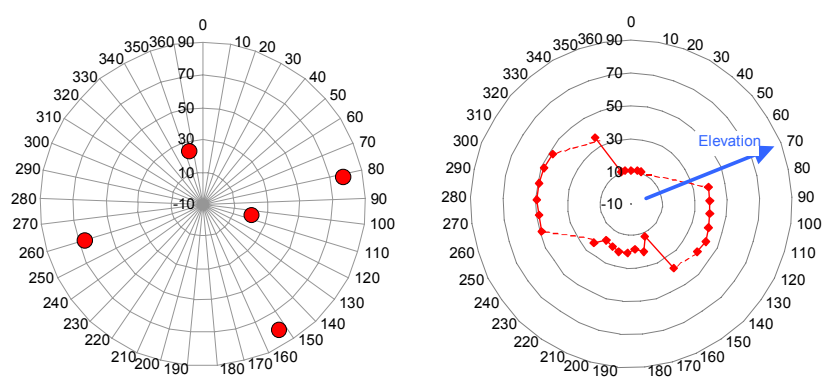

Fig. 3. Schematic representation of the process. 


\section{FIRST EXPERIMENTAL VALIDATION}

Trials were performed along a railway line and in different road environments : urban, suburban, rural. A GPS receiving patch antenna was located on the roof of the vehicle and connected to a Trimble Lassen SK8 receiver to measure the number of satellites received and used for the localization process. A computer managed the acquisitions as a function of time and stored the results.

The number of received GPS satellites and the predicted ones along each trajectory were compared. Whatever the type of environment, the results presented in [20] show generally similar results between predictions and measurements but it appears that the number of satellites taken into account by the GPS receiver for the localization process is generally greater than the number of satellites received only in LOS. Fig. 4 shows a typical result obtained along a railway line. In each environment, the mean difference between the measured and the predicted number of satellites received in LOS is lower than 1.4 (here 0.8). A maximum is observed when the trajectory is surrounded by high obstacles. In these areas, the number of satellites received in LOS is very low. Thus, some of the GPS satellites, situated just below the mask, are received in NLOS and taken into account by the receiver to achieve a localization result.

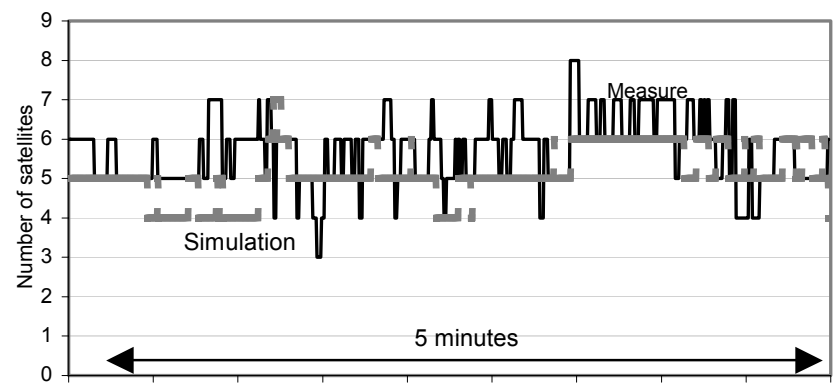

Fig. 4. Example of results : comparison between measurements (gray line) and prediction (black line).

\section{EFFECT OF MULTIPATH}

\section{A. Reception in Non Line-Of-Sight situation}

As already mentioned, the direct visibility and the geometry of the satellites are both parameters that affect the availability of a constellation. Fig. 5 represents the number of received satellites (bold line) and the corresponding PDOP (dotted line) for a given period of time. These curves illustrate that the variations of the PDOP are directly correlated to the variations in the number of available satellites used in the computation.

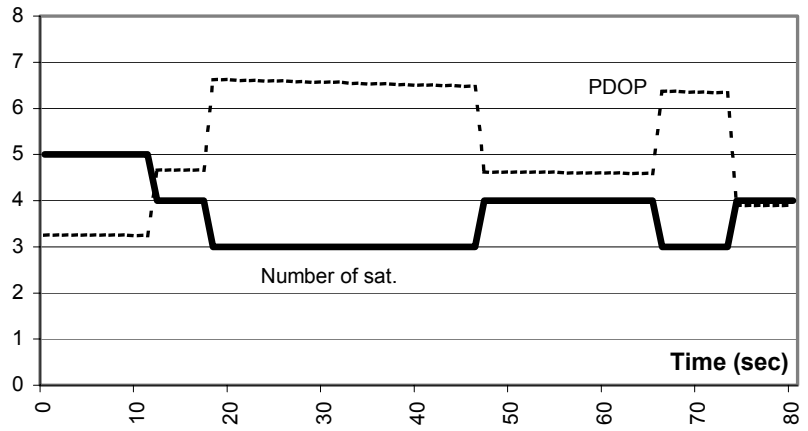

Fig. 5. Evolution of the PDOP with the number of available satellites.

An example of the particular canyon mask is illustrated in Fig. 6 where the bold line represents the optical horizon line at a given position. The other curves show the evolution of the position of several GPS satellites taken into account by the receiver to compute the localization of this point. This figure clearly shows that some satellites are used, even if they are under the optical horizon line. In that case, the satellite signals experience considerable attenuation and introduce an error into the position calculation based on the TOA method. Hence, shadowed satellites must be taken into account to predict the effective availability of the system, and their impact on the error margin must also be quantified.

By analogy with some Land Mobile Satellite channel models [21]-[23] developed for satellite diversity algorithms, the tool has been refined in order to predict which number of satellites are shadowed i.e. received in NLOS, which are used for positioning, and the impact of the NLOS ray on the overall localization accuracy.

To take into account those satellites in the predictive tool, we introduced criteria such as received signal level, PDOP and intrinsic antenna mask elevation. The most restrictive one is the signal-to-noise ratio. Consequently, we have to set up a criterion that will allow the tool to eliminate the satellites that would not be used in the localization process due to their low received signal-to-noise ratio. Both experimental and simulation processes were performed.

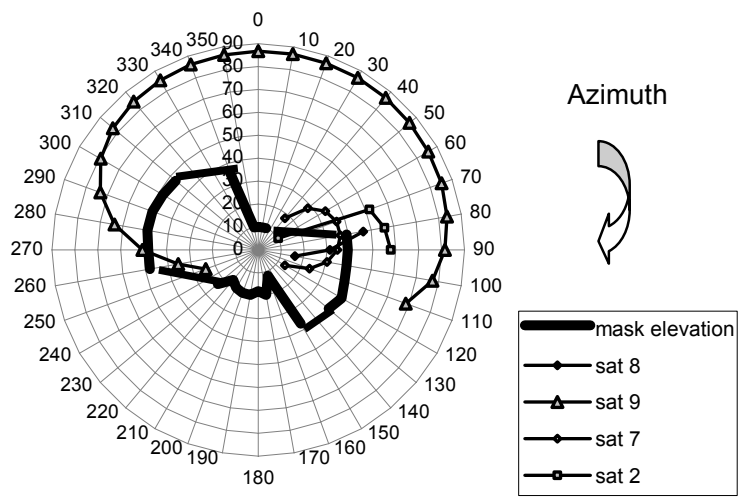

Fig. 6. Position of received satellites around the antenna over several hours. 


\section{B. The Urban canyon configuration}

A GPS antenna was located on the roof of a stationary car, in an urban canyon as drawn in Fig. 7. GPS data such as the PRN (Pseudo Random Number) code and the signal-to-noise $(\mathrm{S} / \mathrm{N})$ ratio of each received satellite were recorded on a computer over several hours.

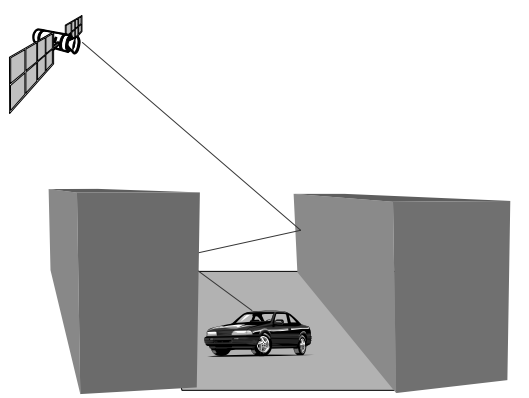

Fig. 7. Illustration of the experimental configuration..

Fig. 8 and Fig. 9 show the S/N ratio in $\mathrm{AMU}^{1}$ (Amplitude Measurement Unit of Trimble ${ }^{\mathrm{TM}}$ ) respectively as a function of the elevation and azimuth of the satellite. A decrease of about 15 AMU in the signal level is measured when the satellite is below the "optical horizon line". It is also possible to classify the $\mathrm{S} / \mathrm{N}$ ratios received respectively from LOS and NLOS satellites using the Rice and Rayleigh distributions [24], as illustrated in Fig. 10. The experimental results have shown the use of multipath signals in the localization computation.

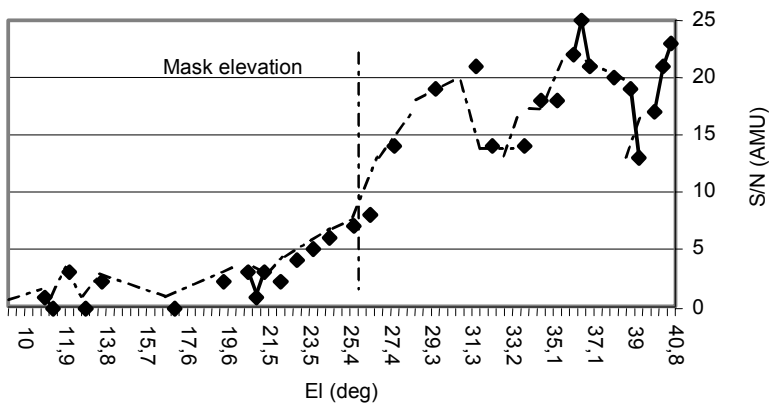

Fig. 8. Received S/N ratio function of the elevation of the satellites.

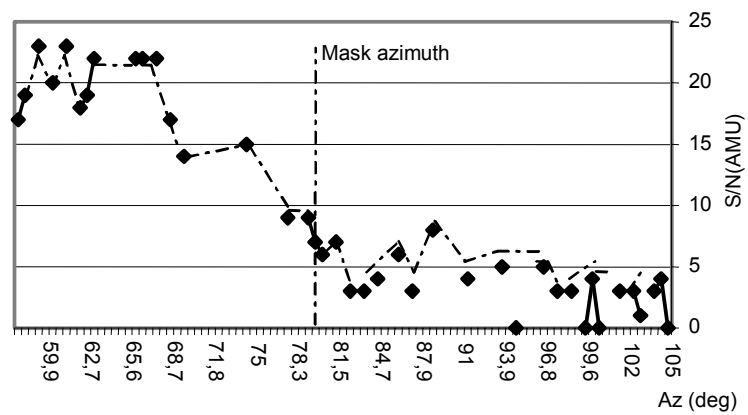

Fig. 9. Received $\mathrm{S} / \mathrm{N}$ ratio function of the azimuth of the satellites.

\footnotetext{
${ }^{1} \mathrm{SNR}(\mathrm{dBHz})=20 \log _{10} \mathrm{AMU}+24$
}

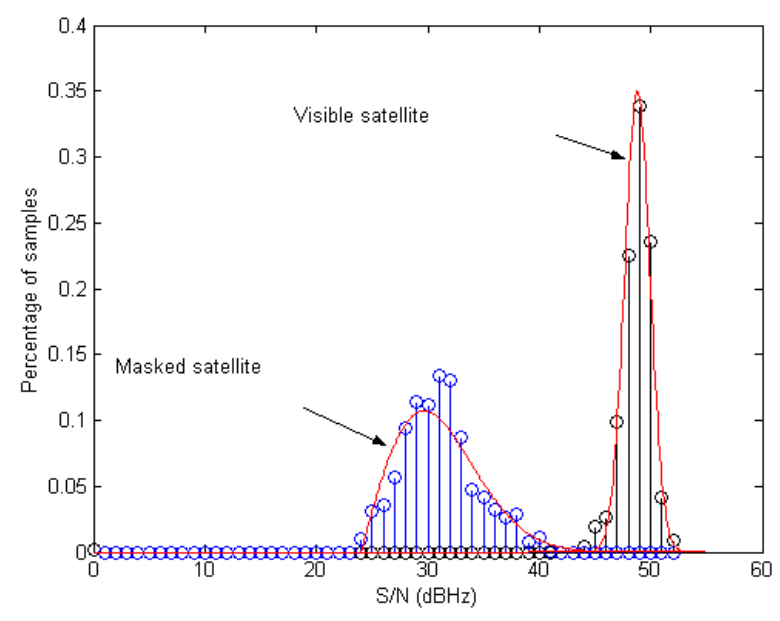

Fig. 10. S/N distributions in LOS and NLOS satellite configurations.

The total received electric field is composed of the sum of the direct, reflected and diffracted contributions as indicated in (1).

$$
E_{\text {total }}=E_{\text {direct }}+E_{\text {reflected }}+E_{\text {diffracted }}
$$

Therefore, in the following, we will study which of these rays is preponderant when there is no direct path received.

1) Diffracted rays: The aim of the simulations performed with a simple model based on the geometrical optical laws, was to separate the contribution of the reflected and diffracted rays on walls and obstacle edges. Fig. 11 gives an example of results obtained as a function of the distance when the receiver is moving along the canyon in the direction of the street. The buildings are represented as regular blocs. The diffraction contribution is very low compared to the reflection contribution.

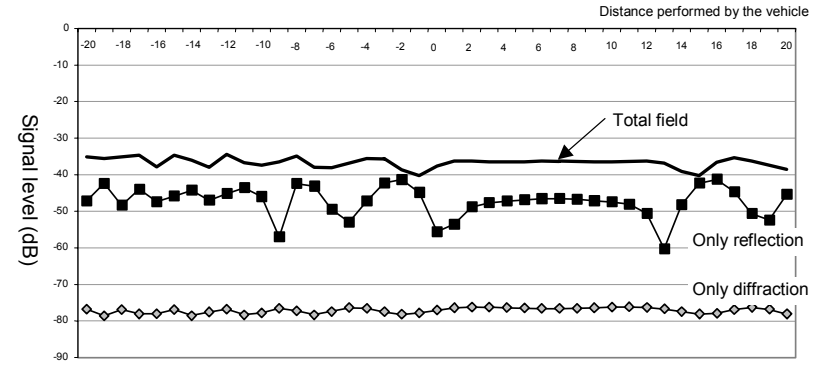

Fig. 11. Respective contributions of reflected and diffracted electric field in the urban canyon configuration.

The diffraction phenomenon leads to high signal attenuation. Thus, the satellites received with diffraction only cannot be used by the GPS receiver. We will consider later the fact that multipath can be identified as one or more reflections.

2) Signal attenuation versus the number of successive reflections :

The reflected electric field can be expressed as a function of the incident electric field and the Fresnel reflection coefficient 
$\Gamma$. The expression of this coefficient depends on the electric field polarization with respect to the reflecting surface. The amplitude of $\Gamma$ varies as a function of the satellite elevation and is lower than, or equal to, 1 [25]. Each reflected path corresponds to a supplementary signal attenuation varying with $\Gamma$. The receiver criterion for satellite signal use is based on the received signal level. We have determined that, in most of the cases, more than one reflection leads to a received signal level lower than the minimum receiver usable signal.

This hypothesis is experimentally verified in a simple urban canyon configuration. Knowing the satellite trajectory, it is possible to calculate the displacement of the point of impact of the second reflection using the simple geometric approach.

Two specific parabolic directive antennas were developed and are connected to an automatic rotation module able to track a chosen GPS satellite along its trajectory. Each antenna offers an $18 \mathrm{dBi}$ gain at $1510 \mathrm{MHz}$. The $3 \mathrm{~dB}$ aperture is $21.2^{\circ}$ and $23.8^{\circ}$ for left and right circular polarization. A view of the experimental radiating pattern in left polarization is given in Fig.12.

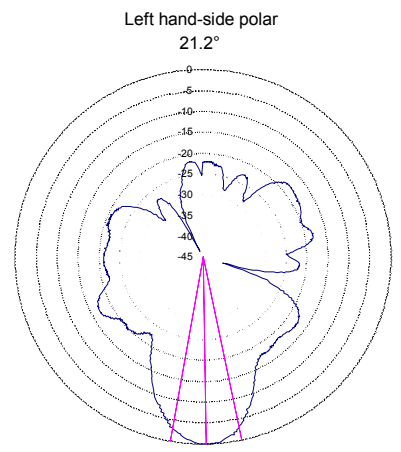

Fig. 12: Experimental radiating pattern in left polarization.

The directive antenna allows us to isolate the reception of a reflected path from a direct path. In the urban canyon configuration, we measured the received signal level of a given GPS satellite chosen according to its trajectory over several days. In Fig. 13, S/N ratios of a direct reception and $\mathrm{S} / \mathrm{N}$ ratios of reception after one reflection function of the elevation of a tracked satellite are compared. To optimize the measurements, we chose the left-hand polarized antenna to receive the direct paths and the right-hand one for the reflected paths. Then, we observe a 4 to $6 \mathrm{dBHz}$ attenuation as shown in Fig. 13.

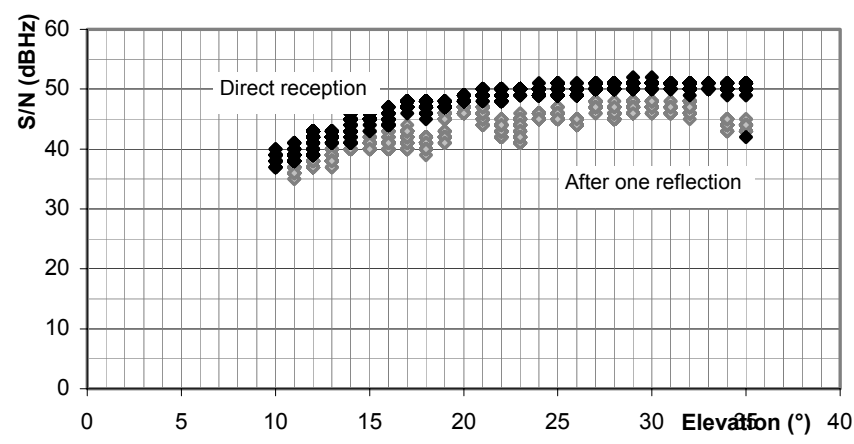

Fig. 13: Comparison of $\mathrm{S} / \mathrm{N}$ as a function of satellite elevation.
In the case of a typical wide beam-width patch antenna used with GPS receivers, a supplementary attenuation is observed due to the wrong polarization rotation, when the signal is received after one reflection on a wall. The circular polarization change effect is illustrated in Fig. 14, when two direct paths are received respectively with the left-hand and right-hand polarized dish illuminating helical antennas.

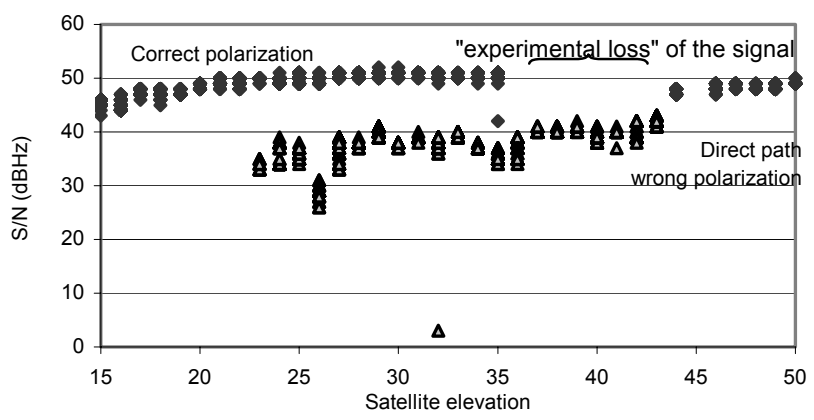

Fig. 14: Depolarization effect on the received signals.

A theoretical and experimental parametric study showed that the obstacle composition does not affect the signal level of the reflected rays [26]. According to this experimental analysis, we enhanced the so-called PREDISSAT tool by including in the prediction the satellites received after only one reflection.

\section{FINAL VERSION OF THE PREDISSAT TOOL}

To include in the prediction the satellites received after one reflection, a second "horizon line" is defined and computed. It represents the minimal satellite elevation angle from which the satellite signal is still received by the GPS receiver, although in NLOS situation. The existence of this second "horizon line" is illustrated in Fig.15, which shows the elevation angle versus time for several satellites in a real urban canyon configuration. The bold line represents the "optical horizon line" corresponding to the top of the buildings. The "horizon line" corresponding to the NLOS reception is drawn as a thin line. In this example, the satellite number 25030 moves from LOS to NLOS configuration. It is shadowed but still used by the GPS receiver when its elevation angle becomes lower than $35^{\circ}$.

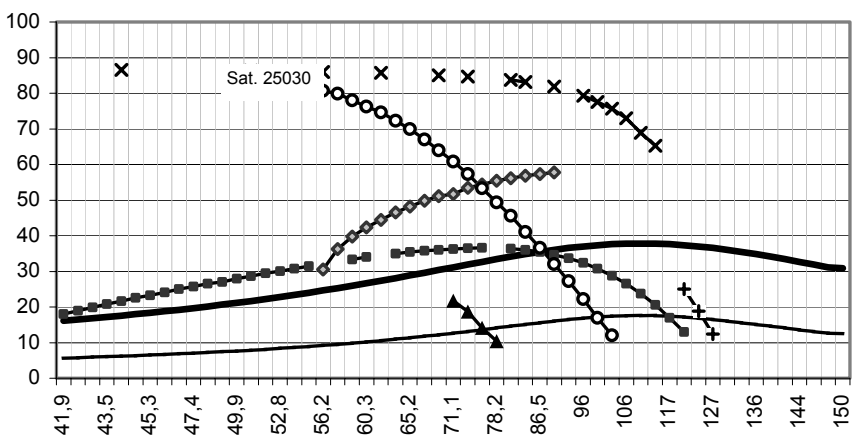

Fig. 15. Received satellites positioned on geometric calculated masks.

The method to define this new "horizon line" is based on a simple geometric approach as indicated in Fig. 16, where a and $\mathrm{b}$ are the perpendicular distances to buildings $\mathrm{B} 1$ and $\mathrm{B} 2$, 
$\mathrm{d}$ the horizontal distance projection of the satellite azimuth, $\mathrm{L}$ the width of the street and N, the direction North. This solution requires the knowledge of distances and heights of the surrounding obstacles. This computation is realised via a mono-camera stereovision process applied on the images previously recorded and described in [26].

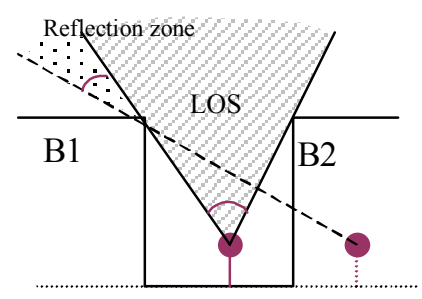

Fig. 16. Geometric representation of the street (front view and view from above).

The lowest elevation for a satellite to be received in LOS, $\mathrm{e}_{\mathrm{LOS}}$ is:

$e_{\text {LOS }}=\arctan \left(\frac{h_{b}-h_{a}}{a}\right)$ for $\mathrm{d}=0$

$e_{L O S}=\arctan \left(\frac{h_{b}-h_{a}}{\sqrt{a^{2}+d^{2}}}\right)$ for $\mathrm{d} \neq 0$

where the distance $d$ is directly linked to the azimuth of the satellite, and $h_{a}$ and $h_{b}$ are the antenna and building heights. The azimuths corresponding to the elevation are :

$A_{B 1}=110+180 \pm \arctan \left(\frac{|d|}{a}\right)$ left side of the street

$A_{B_{2}}=110 \pm \arctan \left(\frac{|d|}{b}\right)$ right side of the street

The lowest elevation angle for a satellite to be received after one reflection $\mathrm{e}_{\mathrm{r}}$ is :

$e_{r}=\arctan \left(\frac{d_{1}}{d_{1}+2 d_{2}} \times \tan \left(e_{L O S}\right)\right)$

where $d_{1}=\sqrt{a^{2}+d^{2}}$ and $d_{2}=\sqrt{b^{2}+d^{2}}$.

The satellite signal will be masked behind building B1 and reflected on building B2.

The dotted line in Fig. 17 corresponds to the lowest elevation of the satellite received after only one reflection. This represents the new horizon line. In this configuration, this limit is situated $10^{\circ}$ to $30^{\circ}$ under the optical line previously considered.

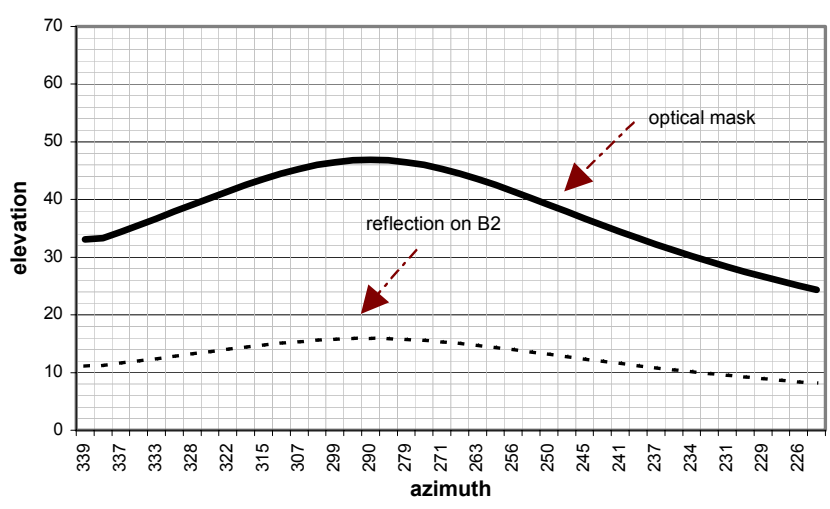

Fig. 17. View of the elevation angle of building B2 and the lowest elevation of reflected signal on it.

This final version of PREDISSAT gives better performances in real urban environment. The trials were performed in the street already presented on Fig. 4. Results are given in Fig 18. The difference between predictions and measurements is considerably reduced. Persistent residual errors are principally results of :

- environmental data extracted from the video that can differ from real environment,

- dynamic characteristics of the receiver we did not implement in the tool.

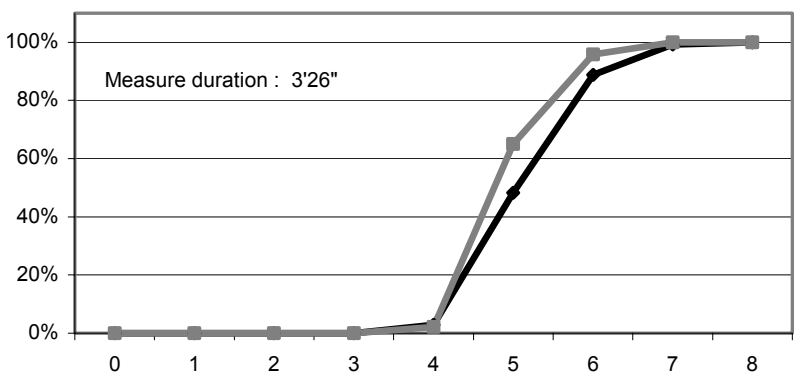

Fig. 18. Example of results : comparison between measurements (gray line) and prediction (black line) as a cumulative distribution function.

\section{ESTIMATION OF THE LOCALISATION ERROR}

Based on the knowledge of the receiving antenna optical environment, it is now possible to evaluate GNSS system availability along a given trajectory in terms of satellite numbers. The use of satellites received after one reflection introduces an error into the pseudo-range estimation. It is now necessary to quantify the influence of this error on the localization process either to discard the satellites or to qualify the position result. The TOA measurement technique will allow us to envisage a very simple method to compute the error introduced by multipath into the localization results as described in [27] and [28]. By assuming that the GPS satellites are far enough from the earth $(20,000 \mathrm{~km})$, we consider that distance $\mathrm{c}$ is equivalent to $\mathrm{d}$ as represented in Fig. 19 and Fig. 20. In this case, it is possible to re-compute the vehicle position. 


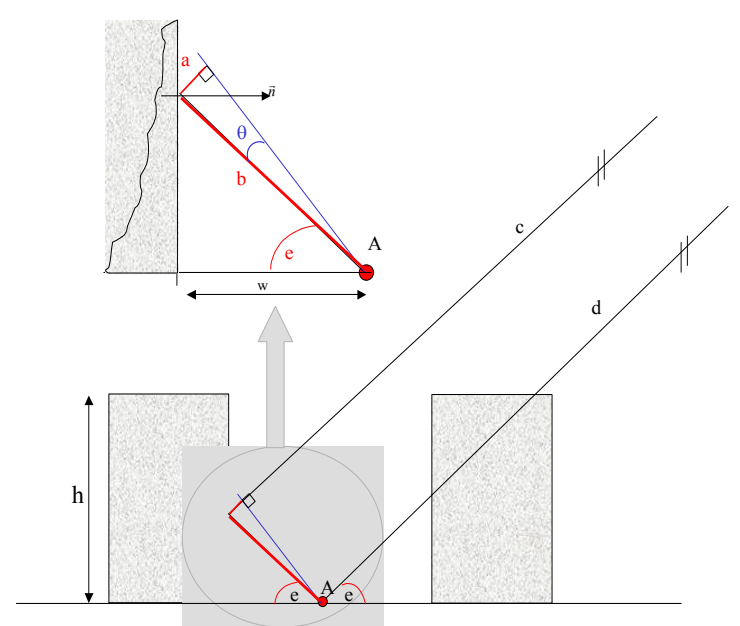

Fig. 19. Calculation principle for pseudo-distance error.

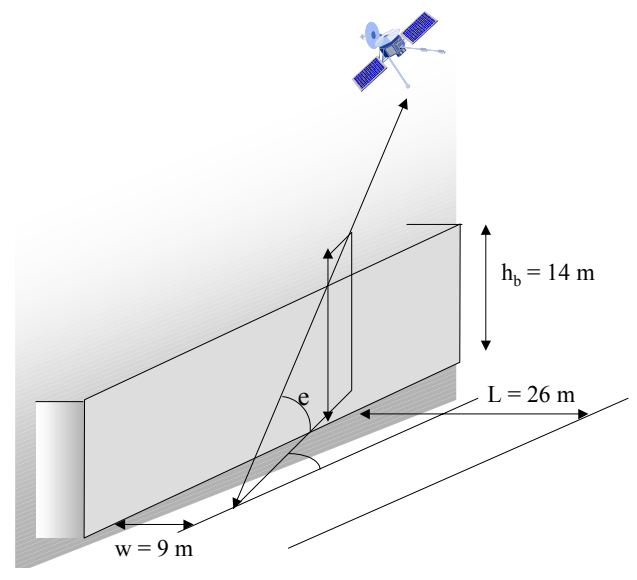

Fig. 20. Assumption schema.

Using this approach, it is possible to compute the pseudorange error $r$ as a function of the satellite elevation e. As an example, we obtain an error $\mathrm{r}=15 \mathrm{~m}$ for a low elevation $\mathrm{e}=$ $35^{\circ}$ and $\mathrm{r}=10 \mathrm{~m}$ for $\mathrm{e}=55^{\circ}$. We introduced then this pseudorange error of $15 \mathrm{~m}$ on the computation process into the case of an urban configuration with a typical mask elevation angle of $30^{\circ}$. Satellites with the lowest elevations will be affected by one reflected path. Thus, we have simulated this error on, successively 2, 3, 4 and 5 satellites among the 6 visible satellites. The results are summed up in the following table 1 .

TABLE 1

LONGITUDE AND LATITUDE ERROR WITH SATELLITES RECEIVED BY MULTIPATH

\begin{tabular}{cl}
\hline \hline $\begin{array}{c}\text { Number of reflected satellites } \\
\text { with a pseudo-range error } \\
\mathrm{r}=15 \mathrm{~m} \text { among the 6 visible }\end{array}$ & Longitude and latitude error \\
\hline 2 & $\mathrm{e}_{\text {Long }}=7 \mathrm{~m}-\mathrm{e}_{\text {Lat }}=11 \mathrm{~m}$ \\
3 & $\mathrm{e}_{\text {Long }}=7 \mathrm{~m}-\mathrm{e}_{\text {Lat }}=11 \mathrm{~m}$ \\
4 & $\mathrm{e}_{\text {Long }}=0 \mathrm{~m}-\mathrm{e}_{\text {Lat }}=22 \mathrm{~m}$ \\
5 & $\mathrm{e}_{\text {Long }}=7 \mathrm{~m}-\mathrm{e}_{\text {Lat }}=22 \mathrm{~m}$ \\
\hline \hline
\end{tabular}

\section{Conclusion ANd Perspectives}

In this paper we have presented an evaluation tool called PREDISSAT, developed to predict a satellite constellation service availability along a given terrestrial trajectory. This tool was developed for GNSS applications and could be used for other non-geostationary earth orbit satellite constellation, as well as for geostationary satellites. It has been shown that it is possible to predict the number of satellites received in LOS and in NLOS configurations after one reflection along a given vehicle trajectory. The process is based on an image processing approach combined with the analysis of satellite signal propagation characteristics and knowledge of the satellite positions within the considered constellation. The tool is used either to construct look-up tables to secure real-time receiver measurements, or to constrain on-board navigation algorithms with the definition of wrong positioning probabilities. PREDISSAT is now under application in the railway field for the development of a new train control and command safe system for low density traffic railway lines within the LOCOPROL (Low Cost satellite based train location system for signalling and train PROtection for Low density traffic railway lines) project of the European Commission.

\section{REFERENCES}

[1] Y. Zhao, Vehicle Location and Navigation Systems, Boston-London : Artech House Publishers, 1997.

[2] C. Drane, C. Rizos, Positioning Systems in Intelligent Transportation Systems, Boston: Artech House, 1997.

[3] M. Berbineau, M. Heddebaut, M. Schroeder, M. Budzwait, "Existing applications in the maritim, road and Air domains," DEUFRAKO - WP2 Final Report, 1998.

[4] D. Galardini, T. Eloranta, M. Berbineau, R. Libbrecht, "Fail-Safe, Innovative, Cost-Effective, Satellite-based Train Protection, Control and Command LOCOPROL," Deliverable 2.2, Former Projects Analysis Report, Aug., 2002.

[5] K. Tysen Mueller, R. Bortins, "Low-Cost GPS Locomotive System for High Speed Rail Applications," in Proc. of KIS 2001, Banff, Canada, June 5-8 2001

[6] Understanding GPS, Principles and applications, Ed. E. D. Kaplan.

[7] D. Mimoun, S. Abbondanza, J.P. Vincent, "Galileo constellation performance in various environments : a sensitivity analysis," in Proc. of ICSSC-19, Toulouse, France, April, 2001.

[8] M. Malicorne, M. Bousquet, V. Calmettes, C. Macabiau, "Effects of masking angle and multipath on Galileo performances in different environments," in Proc. of 8th St Petersburg Conference on Integrated Navigation Systems, Saint Petersburg, Russia, May 28-30, 2001.

[9] C. Meenan, M. Parks, R. Tafazolli, B. Evans, "Availability of 1st Generation Satellite Personal Communication Network Service in Urban Environments," in Proc of IEEE Vehic. Techno. Conf., Ottawa, Canada, May 18-21, 1998.

[10] D. McNeil Mayhew, "Multi-rate Sensor Fusion for GPS Navigation Using Kalman Filtering", Thesis Dept Elec. Eng., Virginia Polytechnic Institute and State University, Blacksburg, Virginia, May, 1999.

[11] C. E. White, D. Bernstein, A.L. Kornhauser, "Some map matching algorithms for personal navigation assistants", Transportation Research Part C 8 (2000) 91-108.

[12] U. Schneider, J. Troelsen, Introducing digital map information into train positioning system : chances and risks, Comprail proceedings, Computers in Railways VII, Comprail 2000, 11-13 Sept. Bologna, Italy, pp 1271-1280.

[13] B. Cai, X. Wang, Train positioning via integration and fusion of GPS and inertial sensors, Comprail proceedings, Computers in Railways VII, Comprail 2000, 11-13 Sept. Bologna, Italy, pp 1217-1226. 
[14] Satellite Tool Kit - Satellite Systems Analysis Software, by Analytical Graphics. Available: http://www.stk.com/

[15] R. Akturan, W.J. Vogel. "Photogrammetric mobile service prediction," Electronic Letters, 31(3), pp 165-166, 1995.

[16] Y. Karasawa, K. Kimura, K. Minamisono, "Analysis of Availability Improvement in LMSS by Means of Satellite Diversity Based on ThreeState Propagation Channel Model," IEEE Trans. On Vehicular Technology, 46(4), Nov. 1997.

[17] A. Jahn, H. Bischl, G. Heiß, "Channel Characterisation for Spread Spectrum Satellite Communications," in Proc. ISSSTA'96 Intern. Symp. On spread spectrum techniques and applications, Mainz, Germany, Sept 22-25, 1996.

[18] E. Lutz, D.M. Dippold, D. Dolainski and W. Papke, "The Land Mobile Satellite Communication Channel, Recording, Statistics, and Channel Model," IEEE Transactions on vehicular technology, vol. 40, n², pp 375-386- May 1991.

[19] J. Marais, B. Meunier, M. Berbineau, «Utilisation du traitement d'images pour l'évaluation de la disponibilité des satellites GPS le long d'une voie de chemin de fer, " in Revue Générale des Chemins de Fer, Numéro spécial Recherche, Juin 1999 (french language).

[20] J. Marais, B. Meunier, M. Berbineau, "Evaluation of GPS availability for Train Positioning along a Railway Line", in Proc. IEEE Vehicular Technology Conference, Boston, Fall 2000.

[21] G.E. Corazza and F. Vatalaro, "A statistical-model for land mobile satellite channels and its application to non-geostationary orbit systems", IEEE Trans. Veh. Technol., vol.43(3), pp 738-42, 1994.

[22] F. Perez Fontan, M.A. Vasquez Castro \& al, "Methodology for the study of environmental effects on Global Navigation Satellite System (GNSS) propagation", Int. Journal of Satellite Communications, vol. 16, pp 1-22, 1998.

[23] C. Loo, "A statistical model for a land mobile satellite link, " IEEE Trans. Veh. Technol., vol. VT-34, pp 122-127, Aug. 1985.

[24] S.R. Saunders, Antennas and Propagation for Wireless Communication Systems, Ed. Wiley, 1999.

[25] T.S. Rappaport, Wireless Communications: Principles and Practice, Prentice Hall; 1995.

[26] J. Marais, «Localisation de mobiles terrestres par satellites. Mise en œuvre d'outils permettant l'analyse de l'influence des conditions de propagation et des effets de masques sur la disponibilité du service offert », Thesis from the University of Lille, 2002 (French language).

[27] S.R. Saunders, B.G. Evans, "Physical model of shadowing probability for land-mobile satellite propagation," Electronics Letters, Aug. 15th , 1996, vol. 32, nº17.

[28] S.S. Peter Wang, Liangchi Hsu, "Improved GPS Location Positioning in NLOS Propagation Environments, " in Proc. AP 2000, Davos, Switzerland, April 9-14, 2000.

Juliette Marais was born on March 26, 1976, in Lille, France. She received an Engineering degree from the Institut Supérieur d'Electronique du Nord in 1997 and a DEA degree from the University of Lille in 1998. From 1998 to 2002, she pursued the $\mathrm{PhD}$. degree at the French National Institute for Transportation and Safety Research (INRETS), on the analysis of the influence of propagation conditions and masking effects on the service availability of satellite localization systems. She received her Ph.D. in July 2002. She joined INRETS in Sept. 2002. She is currently involved in national and European projects dealing with navigation research in the field of terrestrial transport.

Marion Berbineau was born on September 18, 1962, in Toulouse, France. She received an Engineering degree from the Ecole Universitaire d'Ingénieurs de Lille - Informatique, mesures, Automatique, and her Ph.D. from the University of Lille, respectively in 1986 and 1989. She joined the Institut national de Recherche sur la Sécurité dans les Transport in 1989. She is currently a Research Director and has been head of the Laboratoire Ondes et Signaux pour les Transports since April 2002. Her primary fields of interest are electromagnetic propagation in open or confined areas using natural propagation, radiating cables or wave guides, $2 \mathrm{G}$ and $3 \mathrm{G}$ telecommunication systems applied to the field of transport, navigation/localization methods. She is currently involved in national and European projects dealing with communication, navigation and survey in the field of terrestrial transport.
Marc Heddebaut was born on November 26, 1955, in Somain, France. He received an M.Sc. degree and his Ph.D. from the University of Lille France in 1980 and 1983 respectively. He joined the French National Institute for Transportation and Safety Research (INRETS) in 1983 and, since 1988, has been a research director. Since 1979, he has been working in the field of land mobile communication and electromagnetic compatibility. His primary interest are telecommunication systems dedicated to land transport, mobile localization and command control of automated vehicles. 